\title{
Slices Have More Synapses than Perfusion-Fixed Hippocampus from both Young and Mature Rats
}

\author{
Sergei A. Kirov, ${ }^{1}$ Karin E. Sorra, ${ }^{1,2}$ and Kristen M. Harris ${ }^{1,2}$ \\ ${ }^{1}$ Division of Neuroscience in the Department of Neurology, Children's Hospital, and 2Program in Neuroscience, Harvard \\ Medical School, Boston, Massachusetts 02115
}

\begin{abstract}
Hippocampal slices have long been used to investigate properties of synaptic transmission and plasticity. Here, for the first time, synapses in slices have been compared quantitatively with synapses occurring in perfusion-fixed hippocampus, which is presumed to represent the natural in vivo state. Relative to perfusion-fixed hippocampus, a remarkable $40-50 \%$ increase in spine number occurs in adult hippocampal slices, and a $90 \%$ increase occurs in slices from postnatal day 21 rats. Serial EM shows that all of the dendritic spines have normal synapses with presynaptic and postsynaptic elements; however, not all spine types are affected uniformly. Stubby and mushroom spines increase in the adult slices, and thin, mushroom, and branched spines increase in the immature slices. More axonal boutons with multiple synapses occur in the slices,
\end{abstract}

suggesting that the new synapses form on preexisting axonal boutons. The increase in spine and synapse number is evident within a couple of hours after preparing the slices. Once the initial spine induction has occurred, no further change occurs for up to $13 \mathrm{hr}$ in vitro, the longest time investigated. Thus, the spine increase is occurring during a period when there is little or no synaptic activity during the first hour, and the subsequent stabilization in spine synapse numbers is occurring after synaptic activity returns in the slice. These findings suggest that spines form in response to the loss of synaptic activity when slices are removed from the rest of the brain and during the subsequent $1 \mathrm{hr}$ recovery period.

Key words: plasticity; dendritic spines; CA1 pyramidal cell; multiple-synapse boutons; serial electron microscopy
Hippocampal slices are widely used to study cellular mechanisms of synaptic transmission and plasticity (Bliss and Collingridge, 1993; Bindokas et al., 1998; Diamond et al., 1998; Fisahn et al., 1998; Luscher et al., 1998; Nayak et al., 1998). Many approaches have been used to optimize slice health during experimental investigations. Variables that affect slice health include the following: (1) the age of the animal; (2) the specific composition of the life-supporting media; (3) whether the slices are maintained at the interface of media and oxygen or submerged; and (4) the methods of anesthesia, killing, and cutting the slices (for review, see Garthwaite et al., 1980; Reid et al., 1988; Hajos, 1989; Aitken et al., 1995; Lipton et al., 1995). Although much is known about how different physiological or pharmacological parameters affect slices, relatively little is known about whether synapses are altered in slices.

Because of the slow diff usion of glutaraldehyde $(\sim 400 \mu \mathrm{m} / \mathrm{hr})$, the middle of a brain slice remains hypoxic for at least $30 \mathrm{~min}$ during fixation by immersion in mixed aldehydes (Hopwood, 1967). To overcome this limitation, microwave-enhanced fixation was used to speed greatly the diffusion of glutaraldehyde to the

Received Nov. 24, 1998; revised Jan. 26, 1999; accepted Jan. 28, 1999.

This work was supported by National Institutes of Health Grants NS21184 and NS33574, Mental Retardation Research Center Grant P30-HD18655 (K.M.H.), Program in Neuroscience and Division of Medical Sciences, Harvard University, and the Natural Sciences and Engineering Research Council of Canada (K.E.S.). We thank Dr. John Fiala for thoughtful discussions and Drs. John Davis and John Fiala for assistance with the reconstruction system and image analysis in the Image Graphics Laboratory at Children's Hospital. We thank Marcia Feinberg for assistance on the serial electron microscopy and Karen Szumowski for assistance with the figures.

All three authors contributed equally to this work.

Correspondence should be addressed to Dr. Kristen M. Harris, Division of Neuroscience, Enders 260, Children's Hospital, 300 Longwood Avenue, Boston, MA 02115.

Copyright (C) 1999 Society for Neuroscience $\quad 0270-6474 / 99 / 192876-11 \$ 05.00 / 0$ center of the tissue (Login and Dvorak, 1985, 1994). This procedure reveals hippocampal slices that are optimally preserved throughout their depth within seconds after removal from the life-support chamber (Jensen and Harris, 1989). Using this method, synapses have been quantified in well preserved hippocampal slices after undergoing different experimental treatments, such as long-term potentiation (Shepherd and Harris, 1998; Sorra and Harris, 1998).

These earlier studies compared synapses among different hippocampal slices, all of which experienced the same conditions of preparation and maintenance in vitro. What has not been addressed quantitatively is whether synapses in slices are altered relative to their more native state in vivo. For example, during slice preparation, glial, axonal, and dendritic processes are cut. The cut processes release substances (neurotransmitters, growth factors, potassium, etc.) that can be inhibitory, stimulatory, or even toxic to the intact neurons remaining in the slice. Furthermore, all spontaneous background activity is lost when the hippocampus is removed from the rest of the brain.

The purpose of the experiments reported here was to determine whether slices have an altered complement of synapses relative to perfusion-fixed hippocampus. Several parameters were tested. Slices from both young and mature animals were tested. Slices were maintained for varying times in vitro. The brains were perfused in situ with either fixative containing $2.5 \%$ glutaraldehyde, which is standard, or $6 \%$ glutaraldehyde, which matches the concentration used to obtain rapid tissue preservation in the slices.

Serial electron microscopy was used to perform two types of analyses. One analysis used unbiased sampling to compare synapse densities in volumes of hippocampal CA1 neuropil. The other analysis used three-dimensional reconstruction of dendrites 


\begin{tabular}{|c|c|c|c|c|c|c|c|c|c|}
\hline & \multicolumn{4}{|l|}{ Study 1} & \multicolumn{2}{|l|}{ Study 2} & \multicolumn{2}{|l|}{ Study 3} & \multirow[b]{2}{*}{ Totals } \\
\hline & Perfused & $\begin{array}{l}\text { Early } \\
\text { slices }\end{array}$ & $\begin{array}{l}\text { Late slices } \\
\text { (set 1) }\end{array}$ & $\begin{array}{l}\text { Late slices } \\
\text { (set 2) }\end{array}$ & Perfused & $\begin{array}{l}\text { Late } \\
\text { slices }\end{array}$ & Perfused & $\begin{array}{l}\text { Late } \\
\text { slices }\end{array}$ & \\
\hline \multicolumn{10}{|l|}{ Experimental conditions } \\
\hline Animals $(n)$ & 2 & 3 & 5 & 2 & 2 & 2 & 2 & 2 & 20 \\
\hline Age (d) & 39,77 & $60-65$ & $55-63$ & 53,60 & 68,68 & 65,66 & 21,22 & 21,22 & - \\
\hline Weight (gm) & 137,310 & $279-310$ & $246-310$ & 236,279 & 337,411 & 334,355 & 41,55 & 52,61 & - \\
\hline Fixative (\% glutaraldehyde) & 2.5 & 6.0 & 6.0 & 6.0 & 6.0 & 6.0 & 6.0 & 6.0 & - \\
\hline Time in vitro $(\mathrm{hr})$ & - & $1.75-2.4$ & $6-13$ & $4.5-5.75$ & - & $9-10$ & - & $9-10$ & - \\
\hline Number of series & 5 & 4 & 6 & 12 & 2 & 2 & 2 & 2 & 35 \\
\hline Serial sections per series & $27-68$ & $25-58$ & $26-58$ & $33-38$ & $98-106$ & $107-109$ & $112-134$ & $95-112$ & $25-134$ \\
\hline Section Thickness (nm) & $55-80$ & $58-83$ & $59-77$ & $49-71$ & $64-65$ & $50-60$ & $54-65$ & 56 & $49-83$ \\
\hline \multicolumn{10}{|l|}{ ASD analysis } \\
\hline $\mathrm{SA}\left(\mu \mathrm{m}^{2}\right)$ & 1044 & 469 & 715 & - & - & - & - & - & 2228 \\
\hline HNA $\left(\mu \mathrm{m}^{2}\right)$ & 836 & 301 & 595 & - & - & - & - & - & 1732 \\
\hline Total synapses $(n)$ & 465 & 256 & 395 & - & - & - & - & - & 1116 \\
\hline \multicolumn{10}{|l|}{ Dendrite analysis } \\
\hline Dendrites $(n)$ & 52 & - & - & 129 & 16 & 24 & 21 & 12 & 254 \\
\hline Dendrite Diameter $(\mu \mathrm{m})$ & $0.62 \pm 0.016$ & - & - & $0.62 \pm 0.015$ & $0.53 \pm 0.02$ & $0.59 \pm 0.02$ & $0.55 \pm 0.02$ & $0.52 \pm 0.05$ & - \\
\hline Total Length $(\mu \mathrm{m})$ & 155 & - & - & 254 & 107 & 125 & 155 & 68 & 864 \\
\hline Total Spine Synapses $(n)$ & 543 & - & - & 1914 & 376 & 548 & 332 & 270 & 3983 \\
\hline Total Shaft Synapses $(n)$ & 20 & - & - & 63 & 57 & 31 & 44 & 14 & 229 \\
\hline Total Synapses $(n)$ & 563 & - & - & 1977 & 433 & 579 & 376 & 284 & 4212 \\
\hline
\end{tabular}

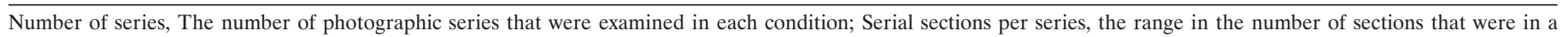
particular series. For example, in Study 1, Late Slices (set 2), there ranged from 33 to 38 serial sections in each of the 12 series.

to compare synapse number per unit length of spiny dendrite. These complementary approaches show that neurons in hippocampal slices have more synapses than in hippocampus fixed by intravascular perfusion at both ages. These findings do not occur from an artifactual decrease in slice volume, because the changes are limited to specific subtypes of synapses.

\section{MATERIALS AND METHODS}

Three studies were performed, as summarized in Table 1. All rats were of the Long-Evans strain, and all of our procedures follow National Institutes of Health guidelines and undergo yearly review by the Animal Care and Use Committee at Children's Hospital. In total, 20 rats were used for these studies.

Perfusion-fixed hippocampus. All perfusion-fixed hippocampus was obtained from animals that were under deep pentobarbital anesthesia (80 $\mathrm{mg} / \mathrm{kg}$ ). Two different preparations of mixed aldehyde fixatives were used. For study 1, the brains from two male rats had been fixed in situ via intravascular perfusion with mixed aldehydes containing $2.5 \%$ glutaral-

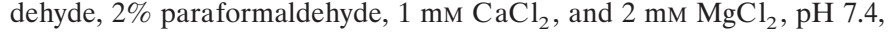
at $40-45^{\circ} \mathrm{C}$ and 4 psi pressure under deep pentobarbital anesthesia (Harris and Stevens, 1989; Harris et al., 1992). For studies 2 and 3, littermates of the four animals used in the slice experiments were perfused through the heart with $5 \mathrm{ml}$ of saline, followed by the same mixed aldehyde solution, except containing $6 \%$ glutaraldehyde to match the concentration used for the slice experiments described below.

Hippocampal slices. Slices were prepared according to standard procedures (Harris and Teyler, 1984; Jackson et al., 1993; Sorra and Harris, 1998). In study 1 , slices were obtained after rapidly killing the animals using a guillotine. For these slices experiments, 10 male rats were used. In studies 2 and 3, the animals were first anesthetized with the same dosage of pentobarbital that was used for the perfusion fixation, before guillotining, to control for any potential artifacts of anesthesia in the perfusion-fixed tissue. In study 1 , two mature, and in study 3 , two immature male rats were used. Four to six $400 \mu \mathrm{m}$ slices from each animal were cut at $70^{\circ}$ transverse to the long axis from the middle third of the hippocampus with a tissue chopper (Stoelting Co., Wood Dale, IL) and placed into ice-cold physiological saline containing $117 \mathrm{~mm} \mathrm{NaCl}, 5.3$ $\mathrm{mm} \mathrm{KCl}, 26 \mathrm{~mm} \mathrm{NaHCO}{ }_{3}, 1 \mathrm{~mm} \mathrm{NaH} \mathrm{PO}_{4}, 2.5 \mathrm{~mm} \mathrm{CaCl} 2,1.3 \mathrm{~mm}$
$\mathrm{MgSO}_{4}$, and $10 \mathrm{~mm}$ glucose, equilibrated with $95 \% \mathrm{O}_{2}-5 \% \mathrm{CO}_{2}, \mathrm{pH} 7.4$. Slices were transferred in this saline via the blunt end of a glass pipette directly onto nets over wells with physiological saline, at the interface of humidified $95 \% \mathrm{O}_{2}-5 \% \mathrm{CO}_{2}$ at $32^{\circ} \mathrm{C}$ in a recording chamber (Stoelting Co.), and maintained for varying times in vitro.

Physiological recordings were done to ensure slice viability (Harris and Teyler, 1984; Jackson et al., 1993; Sorra and Harris, 1998). Two concentric bipolar stimulating electrodes were positioned $600-800 \mu \mathrm{m}$ apart in the middle of stratum radiatum on either side of a single extracellular recording electrode. Slices were judged healthy if the stimulus-response curves were sigmoidal and the half-maximal responses remained stable for at least $1 \mathrm{hr}$ before fixation. At the end of each experiment, the slices were fixed in mixed aldehydes containing $2 \%$ paraformaldehyde, $6 \%$

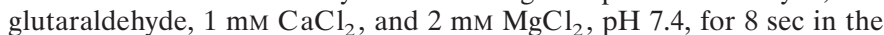
microwave oven, which resulted in a measured final temperature that was always $<50^{\circ} \mathrm{C}$ and usually $<37^{\circ} \mathrm{C}$ to prevent microtubule destruction that occurs above $60^{\circ} \mathrm{C}$ (Jensen and Harris, 1989).

Light and electron microscopy. For study 1, perfusion-fixed tissue and slices were hand processed by routine procedures as described previously (Harris and Stevens, 1989; Harris et al., 1992; Sorra and Harris, 1998). For studies 2 and 3, the fixed tissue was stored overnight in the fixative at room temperature, and then $400 \mu \mathrm{m}$ slices were cut from the perfusionfixed hippocampus embedded in agar and processed with the slices that had been maintained in vitro. All tissue slices were rinsed five times in buffer with repeated agitation. Each slice was manually trimmed under a dissecting microscope to a region containing only area CA1. Slices were soaked briefly in $1 \%$ osmium and $1.5 \%$ potassium ferrocyanide in 100 $\mathrm{mm}$ cacodylate buffer while being cooled in an ice bath until they reached a temperature $<15^{\circ} \mathrm{C}$ and then microwaved for $2.5 \mathrm{~min}$ at $37^{\circ} \mathrm{C}$ using the Pelco 3450 Laboratory Microwave Processor (Ted Pella Inc., Redding, CA). After several buffer rinses, the slices were put into $1 \%$ osmium in $100 \mathrm{~mm}$ cacodylate buffer, cooled, and microwaved for $2.5 \mathrm{~min}$ at $37^{\circ} \mathrm{C}$. Slices were then rinsed four to five times in buffer and twice in water, stained en bloc with $1 \%$ aqueous uranyl acetate while cooled on ice, and microwaved for $2.5 \mathrm{~min}$ at $37^{\circ} \mathrm{C}$, followed by two brief water rinses. Samples were dehydrated in an acetone series $(50,70,90$, and $100 \%)$ for $40 \mathrm{sec}$ each in the microwave oven at $37^{\circ} \mathrm{C}$. Infiltration began with acetone and 1:1 Epon/Spurr's resins for $1 \mathrm{hr}$ on a rotator at $25^{\circ} \mathrm{C}$, followed by 2:1 acetone Epon/Spurr's resins overnight. After replace- 
ment with fresh $100 \%$ resin for several hours, samples were embedded in coffin molds with the dendrites orthogonal to the cutting plane. Samples were cured for $48 \mathrm{hr}$ at $60^{\circ} \mathrm{C}$

The blocks were trimmed to contain a region spanning the width of the slices and in the middle of stratum radiatum midway between area CA3 and the subiculum. Then, several $1-\mu \mathrm{m}$-thick and 60 -nm-thin test sections were taken spanning the full width of the slices. Thick sections were stained with $1 \%$ toluidine blue to guide subsequent trimming. Thin sections were mounted on Pioloform-coated (SPI Supplies, West Chester, PA) slot grids (Synaptek; Ted Pella Inc.) and counter stained with saturated ethanolic uranyl acetate, followed by Reynolds lead citrate, each for $5 \mathrm{~min}$. Sections were examined with a JEOL (Peabody, MA) $1200 \mathrm{EX}$ electron microscope to choose an area midway between the air and net surfaces of the hippocampal slice for subsequent serial thin sectioning. At an optimal depth between 100 and $200 \mu \mathrm{m}$ from the cut surfaces, excellent tissue preservation was found, as evidenced by well preserved dendrites, with intact mitochondria, microtubules, and synapses, and the relative absence of dark or swollen neuronal processes (see Figs. 2-5). A square diamond trimming tool (Electron Microscopy Sciences, Fort Washington, PA) was used to prepare a small trapezoidal area $<100 \mu \mathrm{m}$ on a side for study 1 and $<50 \mu \mathrm{m}$ on a side for studies 2 and 3. Serial thin sections were cut on the Leica (Malvern, PA) Ultracut $\mathrm{S}$ ultramicrotome, mounted, and counter stained as above for the test thins. Individual grids were placed in grid cassettes (Advance Microscopy Techniques, Danvers, MA), stored in numbered gelatin capsules (Electron Microscopy Sciences), and mounted in a rotating stage to obtain uniform orientation of the sections on adjacent grids. All studies were photographed at 150-200 $\mu \mathrm{m}$ from the CA1 pyramidal cell body layer.

The series of sections were photographed at 4000-6000 $\times$ magnification for study 1 perfused, early, and set 1 of the late slices, and at 10,000 $x$ magnification for the second set of late slices in study 1 and all conditions in studies 2 and 3. Calibration grids (Ernest Fullam Inc., Latham, NY) were photographed with each series. In total, $35 \mathrm{EM}$ series were analyzed, ranging from 25 to 134 serial sections (Table 1).

Adjusted synapse density analysis. The adjusted synapse density (ASD) was computed for the conditions of study 1, as outlined in Table 1, using the following equation (for review, see Harris, 1994; Sorra and Harris, 1998):

$$
A S D\left(\# \text { synapses } / 100 \mu m^{3}\right)=\left(n_{\text {syn }} / \mathrm{HNA}\right) * \operatorname{mean}\left(1 / n_{\text {sect }}\right) *(1 / \mathrm{st})
$$

Synapse number $\left(n_{\text {syn }}\right)$ was computed by counting the number of postsynaptic densities (PSDs) occurring within a sample area (SA) or on two of the four lines defining the sample area. Synapses were counted if the PSD was evident on the sample section and if the presynaptic vesicles occurred on the sample section or on the adjacent section for obliquely cut synapses. Because synapse density is markedly influenced by elements occurring nonuniformly in the SA (i.e., myelinated axons, cell bodies, and large dendrites with section profiles $>0.94 \mu \mathrm{m}^{2}$ ), the areas of these elements were measured and subtracted from the sample areas to obtain the homogeneous neuropil area (HNA). Approximately half of each sample field was analyzed for synapses by two investigators.

PSDs have different shapes and sizes, and the probability of capturing them on a single sample section differs in proportion to the number of sections they occupy. Thus, the number of sections each synapse occupied was counted, and the average number of sections $\left(n_{\text {sect }}\right)$ was computed for each synapse type described in Results. The mean inverse of the $n_{\text {sect }}$ per condition was used to adjust for any differences in viewing probability, thereby removing potential size, shape, or orientation biases.

Sampling is also affected differentially by section thickness. Every effort was made to obtain uniform section thickness at the time of cutting (platinum-colored sections in the diamond boat); however, the same section colors are not necessarily the same section thickness (Peachey, 1958). A better estimate of section thickness was obtained for each series by measuring the diameters $(d)$ of longitudinally sectioned mitochondria or dendrites, counting the number of serial sections they occupied $(n)$, and computing section thickness (st) as: $s t=d / n$.

Dendrite analysis. Spine number per unit length of dendrite was computed for lateral apical dendritic segments only (having a diameter of $<1$ $\mu \mathrm{m})$, because the primary apical dendrites were too large and infrequent to obtain useful statistics (Table 1). Furthermore, the primary apical dendrites were far more spiny, and thus could not be grouped with the lateral dendrites (data not shown). Dendritic segment lengths equaled the number of sections they spanned multiplied by section thickness for cross-sectioned dendrites. Segment lengths were computed with the

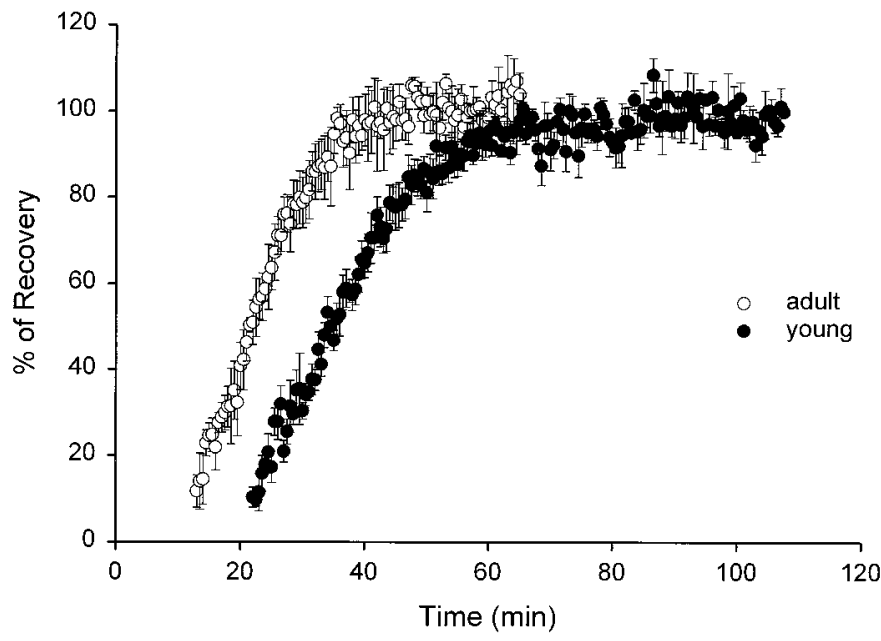

Figure 1. Recovery of the fEPSP after slice dissection. Recording and stimulating electrodes were placed into the slice soon after it was cut and transferred to the recording chamber (time $=0$ ). A constant stimulus intensity of $100 \mu \mathrm{A}$ delivered every $30 \mathrm{sec}$ was used to assess response recovery and stabilization over time. This stimulation intensity was determined a priori to assess slice recovery because field EPSPs were found to be half-maximal at or approximately at a stimulus intensity of $65 \mu \mathrm{A}$. The responses are plotted as the mean \pm SEM of the percent recovery determined relative to the plateau response. Data are averaged from three adults ( 1 from study 1 and 2 from study 2 ; there was no effect of anesthesia on response recovery) and five young animals. Response recovery is delayed by $\sim 10-15$ min at $\mathrm{P} 21$ relative to adults.

Pythagorean theorem by triangulation for obliquely sectioned dendrites (Shepherd and Harris, 1998, their Figure 1).

Statistical analysis. Appropriate statistical tests were used to evaluate the significance of differences between sample populations using SigmaStat (Jandel, San Rafael, CA).

All conditions were analyzed blind as to experimental procedures and were replicated by two or three people.

\section{RESULTS}

\section{Physiological analysis}

The physiological responses were monitored for a couple of hours in slices at both ages, beginning 15-20 min after they were transferred to the recording chamber (Fig. 1). No physiological responses were elicited in slices during the first 10-15 $\mathrm{min}$, regardless of the stimulus intensity delivered and regardless of whether there was anesthesia before guillotining. During the next $30 \mathrm{~min}$, there was a dramatic recovery of the field EPSP (fEPSP), which was stabilized by $\sim 35 \mathrm{~min}$ in adult and by $\sim 1 \mathrm{hr}$ in young hippocampal slices. This recovery phase is typical of all slice experiments, and the data are presented only to emphasize the dramatic physiological events taking place after slicing.

\section{Tissue quality and synapse composition}

Light microscopy revealed well preserved tissue in the CA1 pyramidal cell layer and in stratum radiatum (Fig. 2). Optimal tissue quality occurred in the middle $200 \mu \mathrm{m}$ of the slice (Fig. 3a), which was unchanged up to $13 \mathrm{hr}$ in vitro, the longest time studied in these experiments (Figs. 4, 5). If a particular slice had many dark, degenerating processes, shrunken or highly vacuolated and swollen dendrites, disrupted microtubules, and distended mitochondria, the tissue quality was judged unsuitable for quantitative analysis (Fig. 3b). Excellent tissue preservation was a prerequisite for all samples in the perfusion and slice conditions of these 

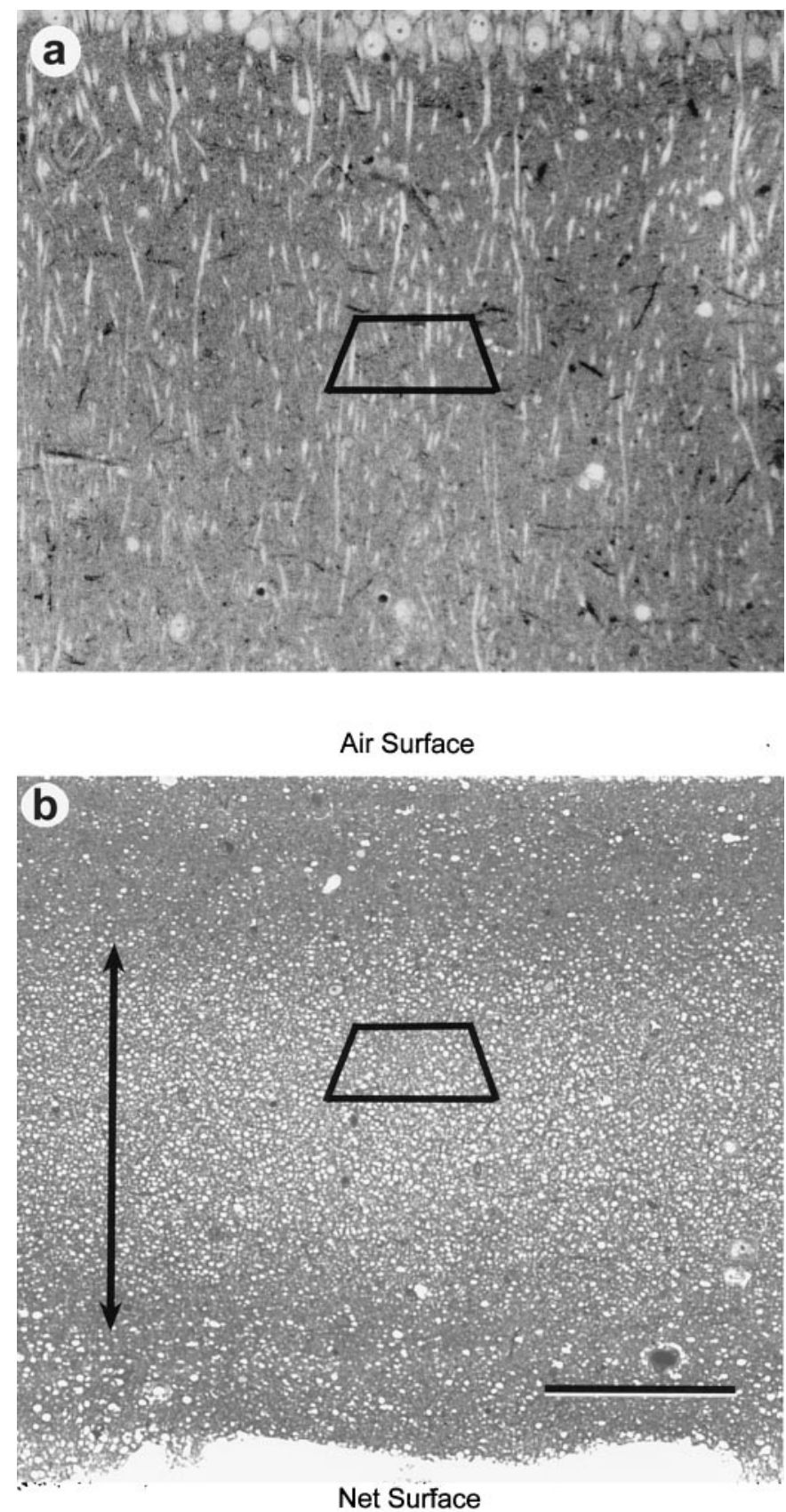

Figure 2. Toluidine blue-stained sections from area CA1 in slices maintained in vitro. $a$, In study 1 , the sections were cut parallel to the long axis of the apical dendritic arbors of the CA1 pyramidal cells for the perfused, the early, and the first set of late slices. $b$, For the 12 series photographed from the second two late slices in study 1 and for all of the slices in studies 2 and 3 , the sections were cut perpendicularly to the apical dendritic arbor to obtain mostly cross-sectioned dendrites, which are optimal for computing the spine number per unit length of dendrite. The air and net surfaces of the slices are labeled, and the double arrow indicates the region of optimal tissue preservation in the middle $200 \mu \mathrm{m}$ of the slice. The trapezoids illustrate the approximate location of subsequent serial thin sections. Scale bar: $a, b, 100 \mu \mathrm{m}$.

experiments (Fig. 4). All synapse and spine types were detected in each condition (Fig. 4) for up to $13 \mathrm{hr}$ (Fig. 5), the latest time evaluated. Dendritic spines were classified as stubby, mushroom, thin, and branched as described in Harris et al. (1992) and Sorra et al. (1998) (Figs. 3-5).

\section{Quantitative analysis of synapses}

The ASDs in the neuropil of perfusion-fixed hippocampus were compared with slices fixed early or late after the onset of incubation in vitro (Table 1 , Study 1 ). The ASD of $213 \pm 17$ synapses/ $100 \mu \mathrm{m}^{3}$ in the perfusion-fixed hippocampus was significantly less than the ASDs of $322 \pm 22$ synapses $/ 100 \mu \mathrm{m}^{3}$ in the early slices and $338 \pm 32$ synapses $/ 100 \mu \mathrm{m}^{3}$ in the late slices $(p<0.02)$ (Fig. $6)$. The ASDs from the early and the late slices did not differ significantly, suggesting that synapse number is maximally elevated by $\sim 2 \mathrm{hr}$ in vitro and then remains stable for the duration (up to $13 \mathrm{hr}$ ).

The results from the ASD analyses were confirmed in the dendrite analysis. The number of synapses per unit length of dendrite was also found to be greater in the slices than in perfusion-fixed hippocampus (Fig. 7). In adult study 1, dendritic segments in the perfused hippocampus had $3.5 \pm 0.2$ spines $/ \mu \mathrm{m}$, in contrast with $4.9 \pm 0.2$ spines $/ \mu \mathrm{m}$ in the slices $(p<0.001)$ (Fig. $7 a$ ). In adult study 2 , the dendritic segments in the perfused hippocampus had $3.5 \pm 0.3$ spines $/ \mu \mathrm{m}$, in contrast with $4.5 \pm 0.2$ spines $/ \mu \mathrm{m}$ in the slices $(p<0.01)$ (Fig. $7 b)$. The difference was even greater at postnatal day 21 (P21), wherein the dendrites in the perfused hippocampus had only $2.2 \pm 0.2 \mathrm{spines} / \mu \mathrm{m}$, in contrast with $4.2 \pm 0.5$ spines $/ \mu \mathrm{m}$ in the slices $(p<0.001)$ (Fig. $7 c)$.

\section{Spine and synapse specificity}

The increase in synapse number in slices was not uniform across different types of dendritic spines. At all ages, thin spines predominated in both the perfused and slice conditions (Fig. 8), and in the adults, there was no significant difference in thin spines between perfused and slice conditions. There were more mushroom and stubby spines (Fig. $8 a, b$ ) and fewer asymmetric shaft synapses in the adult slices (Fig. 8b). At P21, thin, mushroom, and stubby spines occurred in approximately equal proportions in the perfused brain; however, in the slices, there was a dramatic increase in both the thin and mushroom spine categories (Fig. $8 c$ ). Branched spines were rare at all ages, and there was a small significant increase in the slices at P21 only $(p<0.05)$ (Fig. $8 c)$. Additionally, five filopodia were observed at P21 (data not shown).

In studies 2 and 3, synapses were characterized as having macular or perforated PSDs. In the adults, only the macular PSDs increased significantly in slices (Fig. 9a). In contrast, the P21 slices showed a large increase in macular and perforated PSDs (Fig. 9b).

In study 1 , the presynaptic axons associated with every synapse were followed through serial sections to identify whether there was only one PSD [single-synapse bouton (SSB)] or more than one PSD [multiple-synapse bouton (MSB)]. There were more MSBs in the slices $(p<0.03)$ (Fig. 10).

\section{DISCUSSION}

Hippocampal slices have 40-90\% more dendritic spines, depending on age, than comparable regions of hippocampus fixed by perfusion in vivo. The relative increase in dendrite spininess was detected within $2 \mathrm{hr}$ after slicing. Serial EM shows that all of the dendritic spines have normal synapses with presynaptic and postsynaptic elements; however, not all spine types are affected uniformly. Stubby and mushroom spines increase in the adult slices, and thin, mushroom, and branched spines increase in the immature slices. MSBs increase preferentially in the slices, suggesting that the new synapses formed on preexisting axonal boutons. 

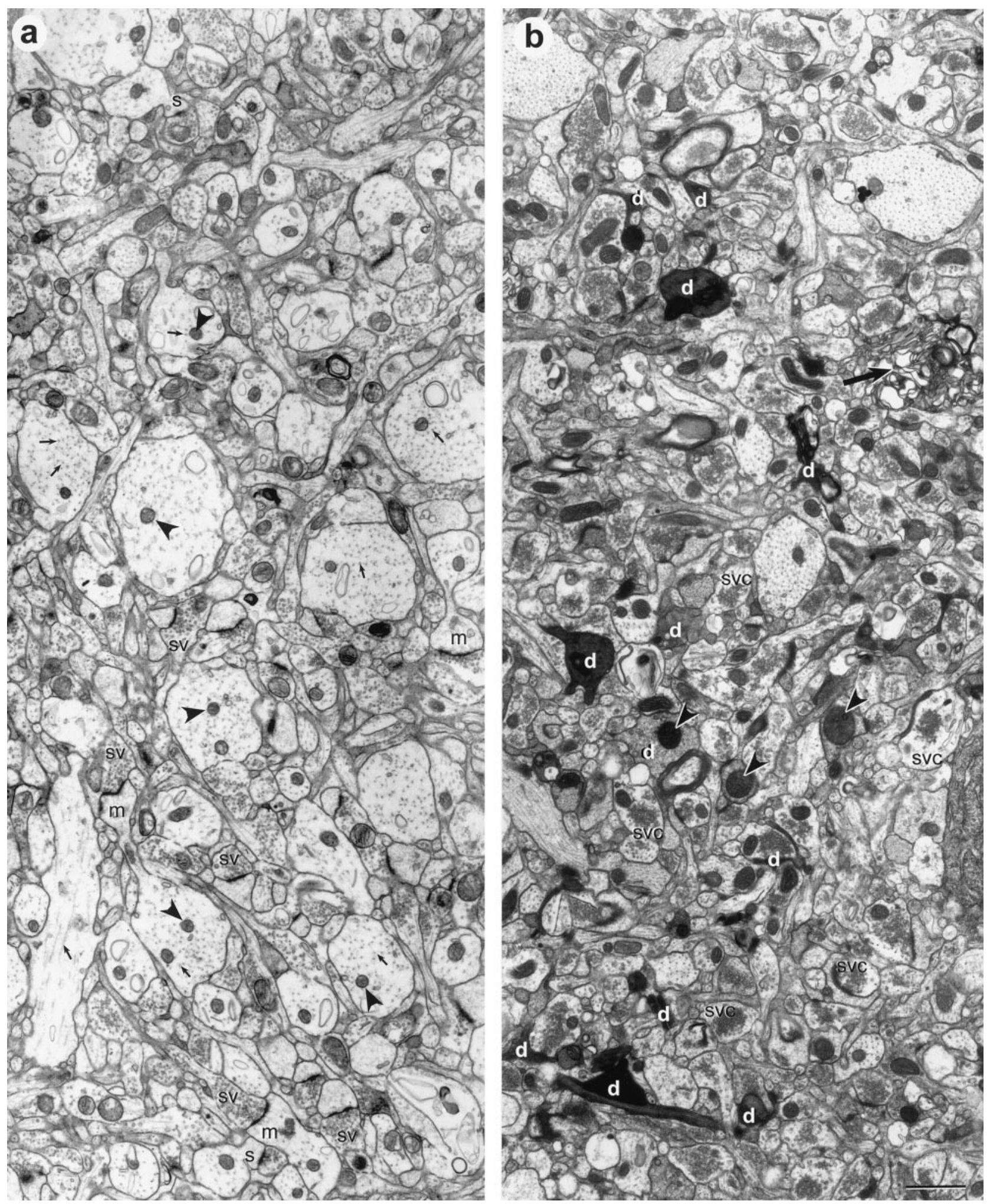

Figure 3. Neuropil from within the middle of stratum radiatum of hippocampal slices. Both slices are photographed at $168-176 \mu \mathrm{m}$ from the air surface of the slice. $a$, In this adult hippocampal slice, the dendrites and axons are well preserved, with distinct microtubules (arrows), clear cytoplasm, nonswollen mitochondria (arrowheads), and a uniform distribution of vesicles in presynaptic axonal boutons (sv). Some examples of longitudinally sectioned mushroom $(\mathrm{m})$ and stubby $(s)$ spines are also evident. $b$, This slice was rejected because of poor tissue quality, as evidenced by numerous shrunken and dark processes $(d)$, swollen mitochondria (arrowheads), regions of unidentifiable whorls of membrane (large arrow), and synaptic vesicular clumping $(s v c)$ in presynaptic axonal boutons. There are some dendrites of good quality interspersed among the degenerating processes; however, it was often difficult to follow the spines past darkened processes, and many of the spines emerging from apparently healthy dendrites synapsed with dark degenerating axons. Scale bar: $a, b, 1 \mu \mathrm{m}$. 

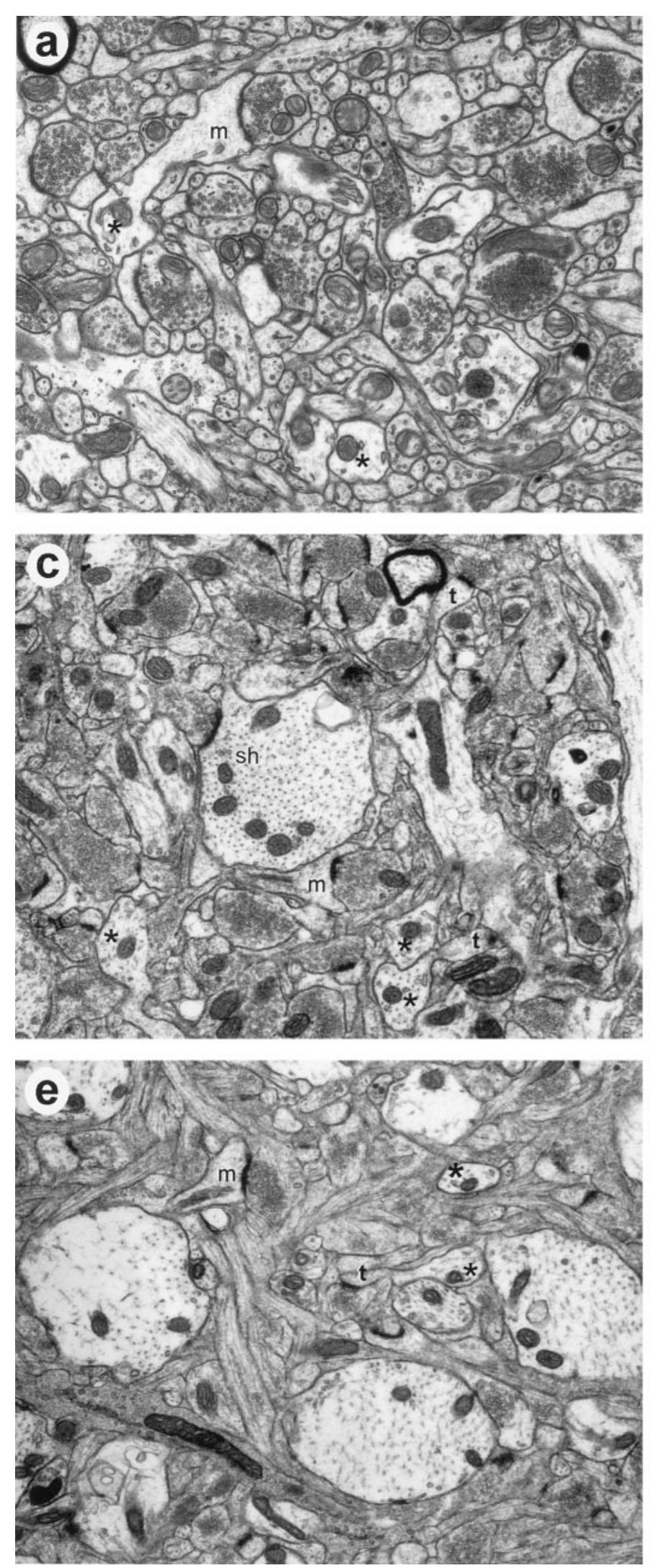
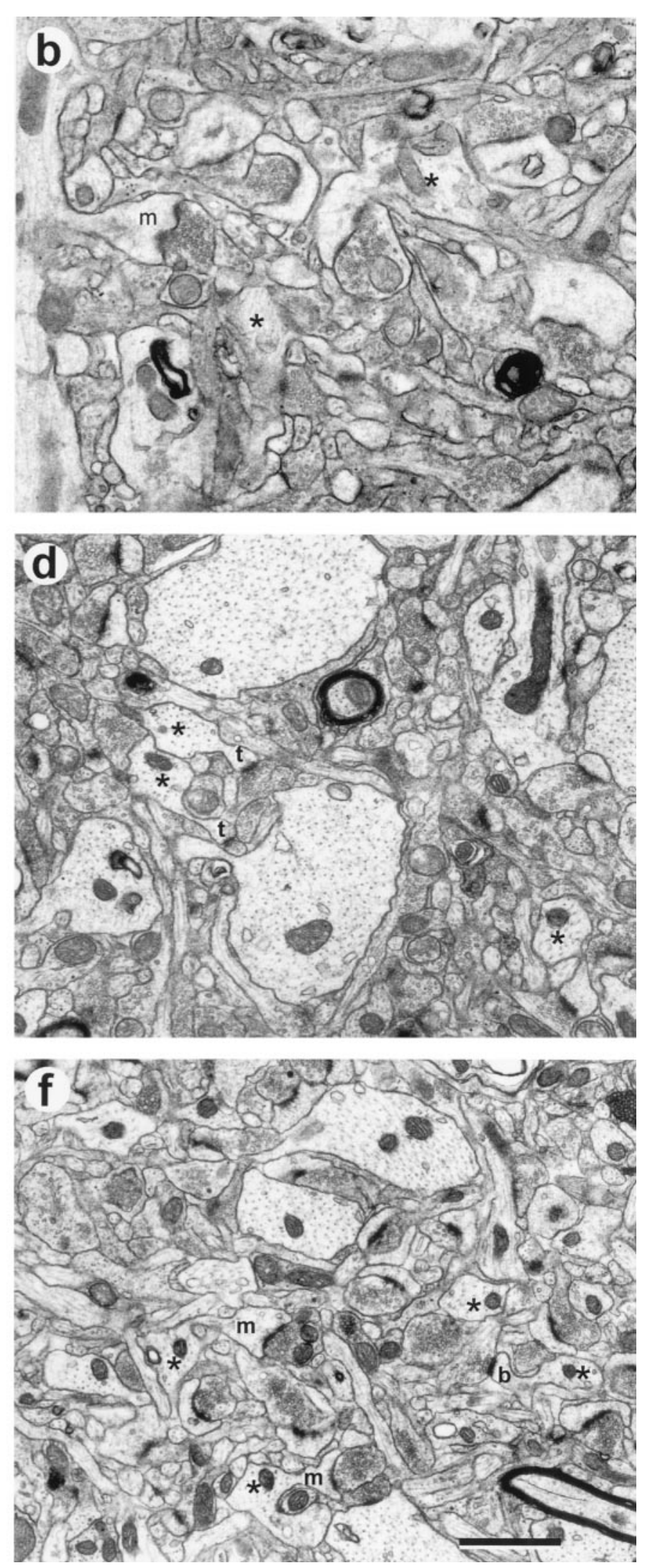

Figure 4. Representative neuropil in the middle of stratum radiatum of area CA1 from each experimental condition. Perfused conditions are on the left, and slice conditions are on the right. $a$, Study 1 , adult hippocampus perfused with $2.5 \%$ glutaraldehyde. $b$, Study 1 , adult early slice. $c$, Study 2 , adult hippocampus perfused with $6 \%$ glutaraldehyde. $d$, Study 2, adult late slice. $e$, Study 3, P21 hippocampus perfused with $6 \%$ glutaraldehyde. $f$, Study 3 , P21 late slice. Sample lateral dendrites are indicated by asterisks. In all cases, the tissue was judged suitable for quantitative analysis of synapses by the overall high quality of tissue preservation. Scale bar: $a-f, 0.5 \mu \mathrm{m}$. 

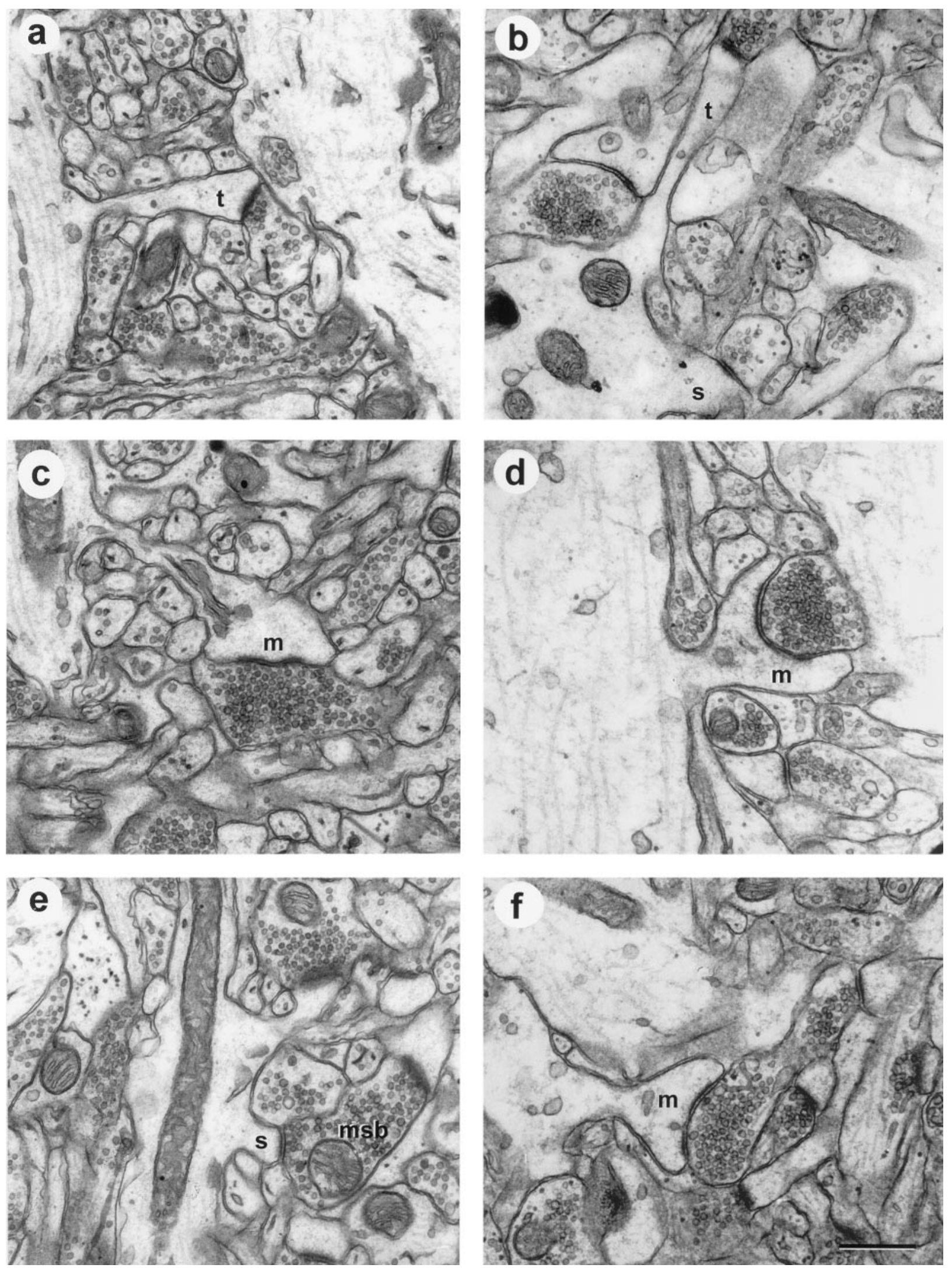

Figure 5. Synapses in area CA1 from perfusion-fixed hippocampus (left) and a hippocampal slice maintained in vitro for 13 hr before fixation (right). These particular examples were selected to illustrate longitudinally sectioned spines, dendrite origins, and presynaptic boutons. $a$, Perfusion-fixed thin spine $(t) . b$, In vitro thin $(t)$ and stubby $(s)$ spines. $c$, Perfusion-fixed mushroom spine. $d$, In vitro mushroom spine. $e$, Perfusion-fixed stubby spine on an MSB. $f$, Another mushroom spine from an in vitro slice. Scale bar: $a-f, 0.5 \mu \mathrm{m}$. 


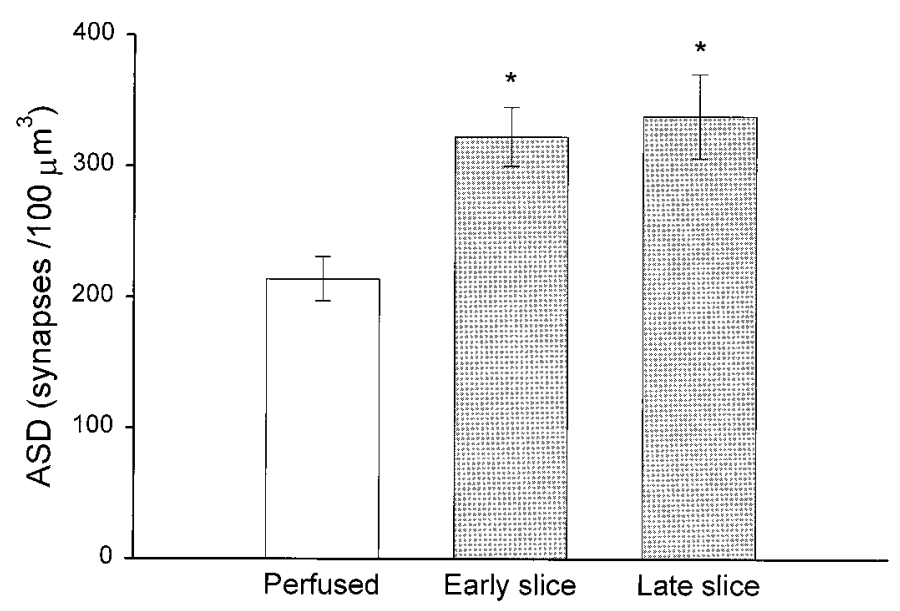

Figure 6. ASD across experimental conditions of adult study 1 (mean \pm SEM). The analysis includes 15 samples from 10 animals, as outlined in Table 1. An overall ANOVA revealed significant differences at $p<0.02$, and the post hoc Tukey test showed that both early and late slices had higher densities than the perfused condition $\left({ }^{*} p<0.05\right)$ and that no significant differences occurred among the early and late slice conditions.

Several potential artifacts can be ruled out as explanations for these findings. Less than $5 \mathrm{~min}$ were needed to prepare the hippocampal slices, and perfusion fixation required 4-5 min of previous anesthesia and $\sim 30 \mathrm{sec}$ before the fixative cleared the brain. Previous studies have shown there are no changes in synapse number with brief periods of hypoxia-ischemia such as these (Hu et al., 1998). Thus, the relative increase in spines is unlikely to result from subtle differences in anoxia during slice preparation versus perfusion fixation. In studies 2 and 3, anesthesia was introduced before slice preparation to control for this potential variable relative to perfusion-fixed brain, and still there were more spines in the slices. Thus, the relative increase in spine number in slices was not, instead, an artifactual loss of spines during anesthesia in the perfusion-fixed brains. All of the slices had a central core of well preserved neuropil (Reid et al., 1988; Jensen and Harris, 1989). This excellent tissue quality, as well as the elevated spine densities, were maintained for at least $13 \mathrm{hr}$ in vitro, the longest time studied.

Another question is whether the slices experienced more shrinkage than in perfusion-fixed brain. If so, this would artificially produce a higher spine density in the slices. Several observations rule against this potential artifact. First, there is a spine and synapse specificity to the effect. If there were a generalized shrinkage in the slices, with no other change, then all types of synapses should be affected uniformly. Wenzel et al. (1994) also found a specific effect of slicing in adult hippocampus, wherein somatic spines were found on dentate granule cells from adult slices, which do not normally occur in hippocampus perfusionfixed in vivo. Second, the dendrite diameters were uniform across conditions, showing that no generalized shrinkage of individual processes occurred (data not shown). Jensen and Harris (1989) also found a constancy in mitochondrial diameters across the slice and perfused conditions. Other studies have found that the extracellular space (ECS) should occupy 20\% of the brain volume (Nicholson and Sykova, 1998); however, some of the ECS is lost during processing of perfusion-fixed tissue, thereby accounting for a net 5-15\% overall tissue shrinkage (Hillman and Deutsch, 1978; Cragg, 1980; Schuz and Palm, 1989). Similarly, EMs from the hippocampal slices show very little extracellular space. The living slices were originally cut at $\sim 400 \mu \mathrm{m}$ thickness, as determined by the Vernier scale on the tissue chopper, but were $\sim 350$ $\mu \mathrm{m}$ thick after processing (Fig. $2 b$ ), accounting for a $12.5 \%$ shrinkage that is comparable to perfusion-fixed tissue.

The ASD analysis was used to obtain an unbiased estimate of synapse densities in the slices and perfused hippocampus. This analysis could be influenced by other factors, such as subtle changes in the density of small glial processes or dendrite diameter, which could alter the relative density of synapses. This potential artifact was minimized by computing the HNA. In addition, the dendrite analysis of spine number per unit length is not sensitive to these potential volume artifacts. Because both approaches revealed the same results, it strengthens the findings from all of the ASD analyses.

The increase in MSBs in slices is consistent with other studies showing that when spines form quickly they make new synapses with preexisting axonal boutons (Woolley et al., 1996). It will also
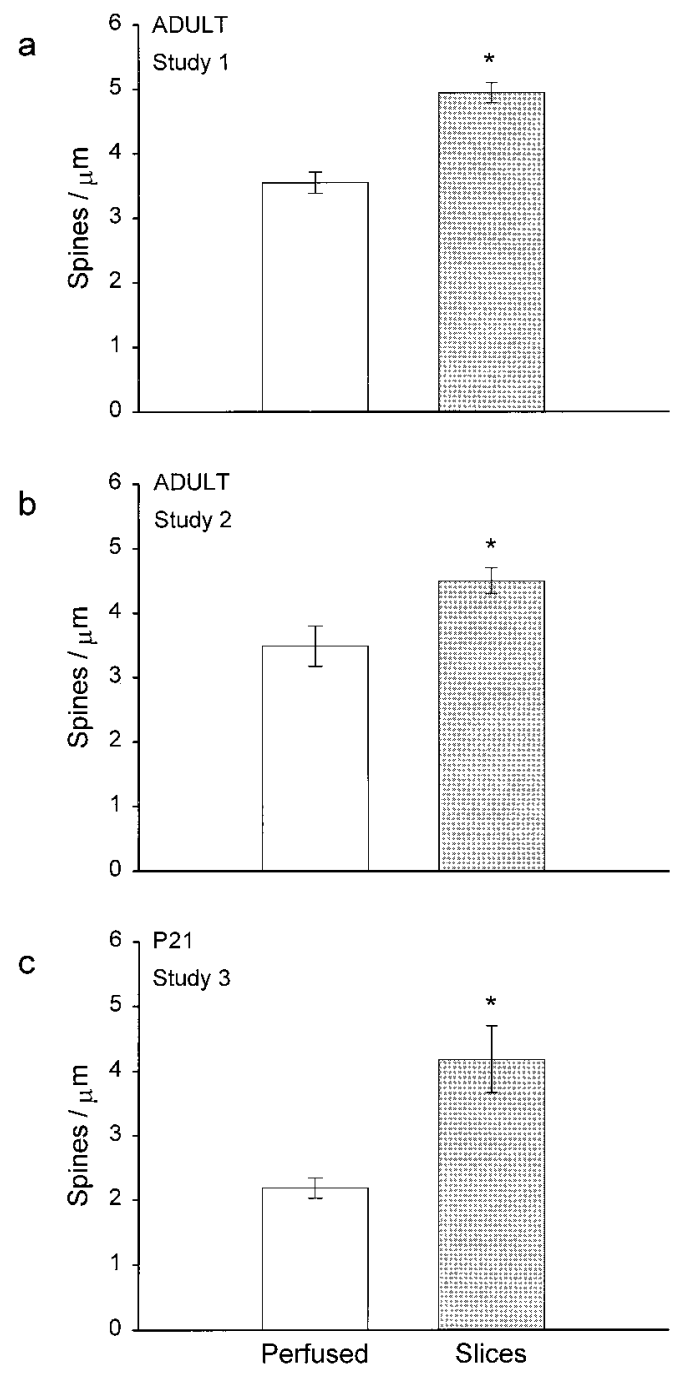

Figure 7. The number of spines per unit micrometer length of dendrite was higher in slices than in perfused hippocampus for all three studies. Data are presented as mean $\pm \mathrm{SEM} ;{ }^{*} p<0.01$, study $1 ;{ }^{*} p<0.001$, studies 2 and 3. See Table 1 for the number of dendrites analyzed in each study. 

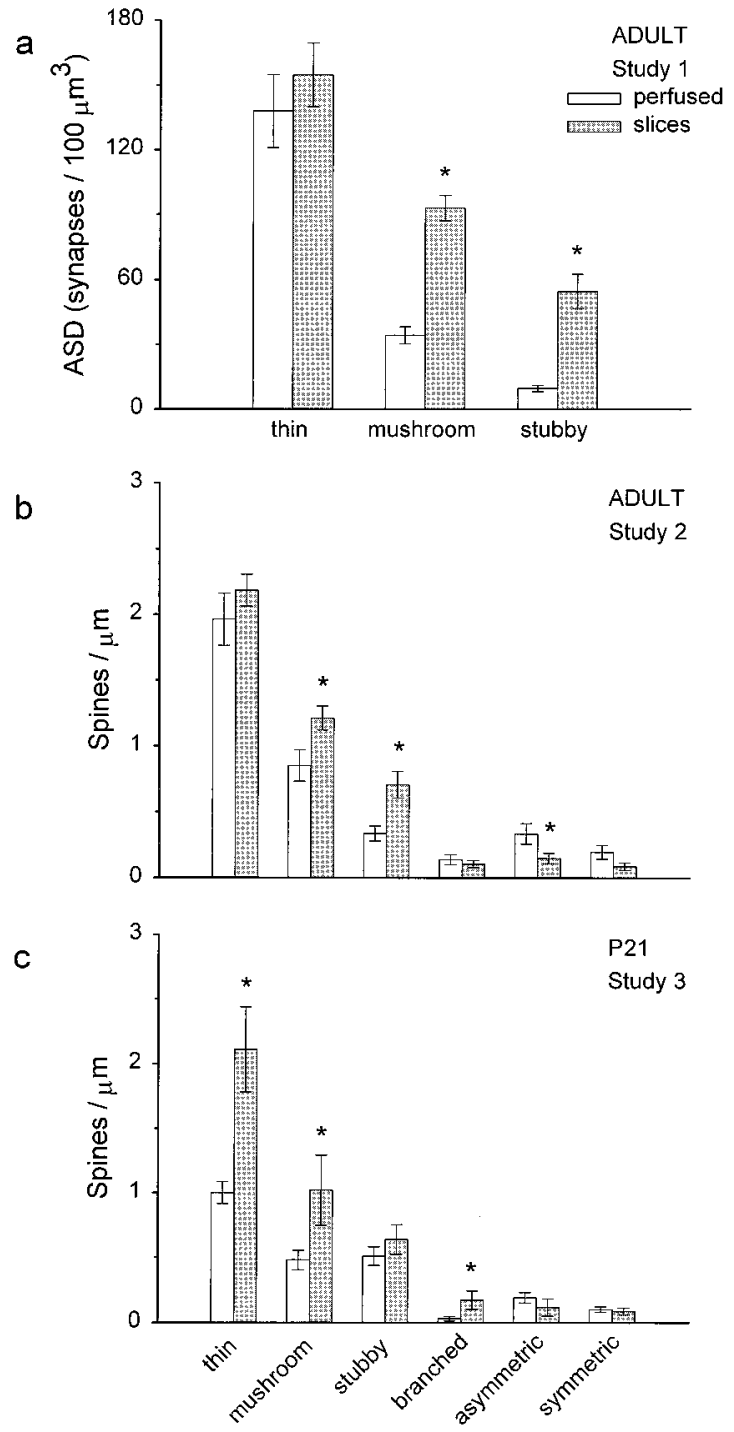

Figure 8. The increase in synapse density affected only some synapse populations. For the adult study 1, only thin, mushroom, and stubby classes were distinguished. In studies 2 and 3, the rare branched spines and two classes of shaft synapse (asymmetric, presumably excitatory; and symmetric, presumably inhibitory) were identified, as well. In all studies, spine synapses that were incomplete within the series accounted for $<10 \%$ of the total synapse number. $a, b$, Thin spines predominated and were comparable across the perfusion and slice conditions $(p=0.43)$, whereas more mushroom $\left({ }^{*} p<0.005\right)$ and more stubby $\left({ }^{*} p<0.005\right)$ spines occurred in slices from adult studies 1 and 2 . In addition, there was a small significant decrease in shaft synapses $\left({ }^{*} p<0.05\right) . c$, There were more thin $\left({ }^{*} p<0.001\right)$, mushroom $\left({ }^{*} p<0.02\right)$, and branched $\left({ }^{*} p<0.03\right)$ spines in slices at P21. Data are presented as mean \pm SEM.

be interesting to learn whether there is a significant portion of nonsynaptic boutons in perfusion-fixed brain that could be the source of the nonsignificant trend toward an increase also in the SSBs.

The increase in stubby spines in the adult slice preparations recapitulates processes that occur during hippocampal development (Harris and Stevens, 1989; Harris et al., 1992; Fiala et al., 1998). During the first postnatal week, hippocampal synapses are recruited to dendritic shafts via filopodia. Then, shaft synapses give rise to stubby spines, which predominate for the next few weeks. As the animals mature, the shaft and stubby spine syn- apses are markedly reduced while thin and mushroom spines are formed, and eventually the thin spines become the dominant spine shape. Curiously, in slices from P21, the thin and mushroom spines reach numbers comparable with those found in the mature hippocampus in vivo and in vitro. These findings emphasize that spine induction is more profound in the developing slices than in mature slices, possibly accelerating an ongoing developmental process.

Together, these arguments support the conclusion that new spines are formed in hippocampal slices. One possible mechanism is that spines are induced by excessive synaptic activation when glutamate and potassium ions are released from the cut processes. This possibility seems unlikely because recent findings show that many more spines are induced when synaptic transmission is completely blocked in slices (Kirov and Harris, 1998). These findings are consistent with observations from developing cortex, which show that blocking synaptic transmission results in spinier developing dendrites (Dalva et al., 1994; Rocha and Sur, 1995). Conversely, excessive activation caused by elevated NMDA results in spine loss (Segal, 1995; Halpain et al., 1998). The spine induction observed in our experiments occurs within the first $2 \mathrm{hr}$, pointing to the loss of spontaneous synaptic activity when slices are removed from the rest of the brain and the "synaptically silent" recovery period as the candidate mechanisms for induction of new spines in slices.

There are several ways in which the new spines could form. One possibility that has been widely suggested, without convincing morphological evidence, is that new spines form from the splitting of previously existing spine synapses. This process is unlikely because branched or "splitting" spine heads never share the same presynaptic axon in hippocampal area CA1 in vivo or in slices
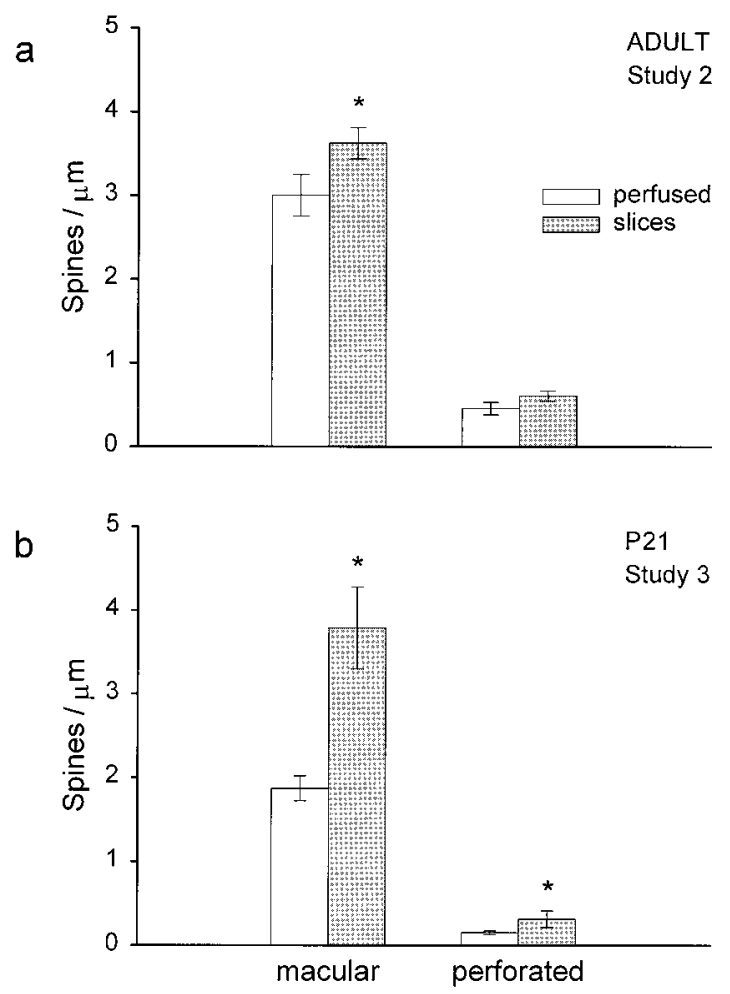

Figure 9. $a$, Macular synapses were greater in adult slices $\left({ }^{*} p=0.05\right) . b$, P21 slices had more macular $\left({ }^{*} p<0.001\right)$ and more perforated $(* p<$ $0.05)$ synapses. Data are presented as mean \pm SEM. 


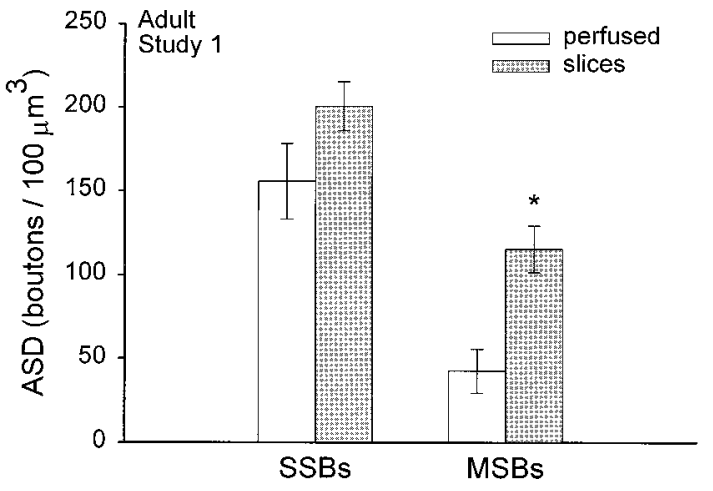

Figure 10. Comparison of the relative incidence of SSBs and MSBs. The incidence of SSBs was not statistically different between the perfused and slice conditions $(p=0.18)$. More MSBs occurred in slices $\left({ }^{*} p<0.03\right)$. Data are presented as mean \pm SEM.

(Sorra et al., 1998). Another possibility is that spines form in response to sprouting axons that are replacing the cut and degenerating processes. This possibility also seems unlikely because degeneration, removal, and sprouting of new axons requires several days, and even then synapse number never reaches the normal predeafferentation level (Wheal et al., 1998). Rapid spine formation in the slices might recapitulate development, namely that after quieting of the neuron, filopodia are induced that guide axons to the dendritic shafts, and spines arise from those shaft synapses. If this is the process, then we would expect to see an increase in shaft synapses and filopodia at an earlier time point (such as within $30 \mathrm{~min}$ after preparing the slices). Some evidence for this process was detected in the P21 slices in which a few filopodia were encountered, even at 9-10 hr after preparing the slices, whereas none were detected in the perfused hippocampus at this age. Alternatively, spine formation in slices may start to recapitulate development in that protrusions are extended, but because the compact neuropil is full of axonal boutons at both P21 and in adult slices, the protrusions immediately encounter good candidates with which to form synapses. This contrasts with early development (P1-P6), wherein filopodia traverse a much looser neuropil that has very few axonal varicosities containing vesicles (Fiala et al., 1998). Because many of the more mature axons already have other synapses on them (i.e., the MSBs) demonstrating their suitability for making synapses, the protrusions develop immediately into dendritic spines without the intermediate stage involving retraction to the dendritic shaft. Further experiments will be needed to assess whether there are intermediate stages in spine formation during the $1 \mathrm{hr}$ recovery period.

In summary, dendritic spines are remarkably plastic in both adult and immature hippocampus as demonstrated here by their profound induction in hippocampal slices. The most likely explanation for this process is that more spines form because the slice is relatively quiet in terms of overall synaptic activity. Once the initial spine induction has occurred during the recovery period, spine and synapse numbers stabilize at the elevated level when synaptic activity resumes in slices maintained for longer periods in vitro.

\section{REFERENCES}

Aitken PG, Breese GR, Dudek FF, Edwards F, Espanol MT, Larkman PM, Lipton P, Newman GC, Nowak Jr TS, Panizzon KL (1995) Pre- parative methods for brain slices: a discussion. J Neurosci Methods 59:139-149.

Bindokas VP, Lee CC, Colmers WF, Miller RJ (1998) Changes in mitochondrial function resulting from synaptic activity in the rat hippocampal slice. J Neurosci 18:4570-4587.

Bliss TVP, Collingridge GL (1993) A synaptic model of memory: longterm potentiation in the hippocampus. Nature 361:31-39.

Cragg B (1980) Preservation of extracellular space during fixation of the brain for electron microscopy. Tissue Cell 12:63-72.

Dalva MB, Ghosh A, Shatz CJ (1994) Independent control of dendritic and axonal form in the developing lateral geniculate nucleus. J Neurosci 14:3588-3602.

Diamond JS, Bergles DE, Jahr CE (1998) Glutamate release monitored with astrocyte transporter currents during LTP. Neuron 21:425-433.

Fiala JC, Feinberg M, Popov V, Harris KM (1998) Synaptogenesis via dendritic filopodia in developing hippocampal area CA1. J Neurosci 18:8900-8911.

Fisahn A, Pike FG, Buhl EH, Paulsen O (1998) Cholinergic induction of network oscillations at $40 \mathrm{~Hz}$ in the hippocampus in vitro. Nature 394:186-189.

Garthwaite J, Woodhams PL, Collins MJ, Balazs R (1980) A morphological study of incubated slices of rat cerebellum in relation to postnatal age. Dev Neurosci 3:90-99.

Hajos F, Garthwaite J, Garthwaite G, Csillag A (1989) Morphology of supravital brain slices pre-incubated in a physiological solution prior to fixation. Acta Morphol Hung 37:181-199.

Halpain S, Hipolito A, Saffer L (1998) Regulation of F-actin stability in dendritic spines by glutamate receptors and calcineurin. J Neurosci 18:9835-9844.

Harris KM (1994) Serial electron microscopy as an alternative or complement to confocal microscopy for the study of synapses and dendritic spines in the central nervous system. In: Three-dimensional confocal microscopy: volume investigation of biological specimens (Stevens JK, Mills LR, Trogadis JE, eds), pp 421-445. New York: Academic.

Harris KM, Stevens JK (1989) Dendritic spines of CA1 pyramidal cells in the rat hippocampus: serial electron microscopy with reference to their biophysical characteristics. J Neurosci 9:2982-2997.

Harris KM, Teyler TJ (1984) Developmental onset of long-term potentiation in area CA1 of the rat hippocampus. J Physiol (Lond) 346:27-48.

Harris KM, Jensen FE, Tsao B (1992) Three-dimensional structure of dendritic spines and synapses in rat hippocampus (CA1) at postnatal day 15 and adult ages: implications for the maturation of synaptic physiology and long-term potentiation. J Neurosci 12:2685-2705.

Hillman H, Deutsch K (1978) Area changes in slices of rat brain during preparation for histology or electron microscopy. J Microsc 114:77-84.

Hopwood D (1967) Some aspects of fixation with glutaraldehyde. J Anat 101:83-92.

Hu BR, Park M, Martone ME, Fischer WH, Ellisman MH, Zivin JA (1998) Assembly of proteins to postsynaptic densities after transient cerebral ischemia. J Neurosci 18:625-633.

Jackson PS, Suppes T, Harris KM (1993) Stereotypical changes in the pattern and duration of long-term potentiation at postnatal days 11 and 15 in the rat hippocampus. J Neurophysiol 70:1412-1419.

Jensen FE, Harris KM (1989) Preservation of neuronal ultrastructure in hippocampal slices using rapid microwave-enhanced fixation. J Neurosci Methods 29:217-230.

Kirov SA, Harris KM (1998) Blocking synaptic transmission in adult rat hippocampal slices induces spine-like protrusions. Soc Neurosci Abstr 24:274.

Lipton P, Aitken PG, Dudek FE, Eskessen K, Espanol MT, Ferchmin PA, Kelly JB, Kreisman NR, Landfield PW, Larkman PM (1995) Making the best of brain slices: comparing preparative methods. J Neurosci Methods 59:151-156.

Login GR, Dvorak AM (1985) Microwave energy fixation for electron microscopy. Am J Pathol 120:230-243.

Login GR, Dvorak AM (1994) Application of microwave fixation techniques in pathology to neuroscience studies: a review. J Neurosci Methods 55:173-182.

Luscher C, Malenka RC, Nicoll RA (1998) Monitoring glutamate release during LTP with glial transporter currents. Neuron 21:435-441.

Nayak A, Zastrow DJ, Lickteig R, Zahniser NR, Browning MD (1998) 
Maintenance of late-phase LTP is accompanied by PKA-dependent increase in AMPA receptor synthesis. Nature 394:680-683.

Nicholson C, Sykova E (1998) Extracellular space structure revealed by diffusion analysis. Trends Neurosci 21:207-215.

Peachey LD (1958) Thin sections. I. A study of section thickness and physical distortion produced during microtomy. J Biophys Biochem Cytol 4:233-245.

Reid KH, Edmonds Jr HL, Schurr A, Tseng MT, West CA (1988) Pitfalls in the use of brain slices. Prog Neurobiol 31:1-18.

Rocha M, Sur M (1995) Rapid acquisition of dendritic spines by visual thalamic neurons after blockade of $N$-methyl-D-aspartate receptors. Proc Natl Acad Sci USA 92:8026-8030.

Schuz A, Palm G (1989) Density of neurons and synapses in the cerebral cortex of the mouse. J Comp Neurol 286:442-455.

Segal M (1995) Morphological alterations in dendritic spines of rat hippocampal neurons exposed to $N$-methyl-D-aspartate. Neurosci Lett 193:73-76.

Shepherd GMG, Harris KM (1998) Three-dimensional structure and composition of CA3 $\rightarrow$ CA1 axons in rat hippocampal slices: implica- tions for presynaptic connectivity and compartmentalization. J Neurosci $18: 8300-8310$.

Sorra KE, Harris KM (1998) Stability in synapse number and size at 2 $\mathrm{hr}$ after long-term potentiation in hippocampal area CA1. J Neurosci 18:658-671.

Sorra KE, Fiala JC, Harris KM (1998) Critical assessment of the involvement of perforations, spinules, and spine branching in hippocampal synapse formation. J Comp Neurol 398:225-240.

Wenzel J, Otani S, Desmond NL, Levy WB (1994) Rapid development of somatic spines in stratum granulosum of the adult hippocampus in vitro. Brain Res 656:127-134.

Wheal HV, Chen Y, Mitchell J, Schachner M, Wieland H, Van Rossum D, Kirsch J (1998) Molecular mechanisms that underlie structural and functional changes at the postsynaptic membrane during synaptic plasticity. Prog Neurobiol 55:611-640.

Woolley CS, Wenzel HJ, Schwartzkroin PA (1996) Estradiol increases the frequency of multiple synapse boutons in the hippocampal CA1 region of the adult female rat. J Comp Neurol 373:108-117. 